\title{
Durch ein Tablet gestützte Biografiearbeit bei älteren Menschen im Pflegeheim: Eine Interview-Studie mit Pflegenden
}

\author{
Christian Buhtz $\cdot$ Denny Paulicke ${ }^{1,2} \cdot$ Mary Wolf $^{3} \cdot$ Sebastian Hofstetter ${ }^{1} \cdot$ Karsten Schwarz $^{1}$ \\ Eingegangen: 29. Januar 2020 / Angenommen: 14. September 2020 / Online publiziert: 6. Oktober 2020 \\ ○ Der/die Autor(en) 2020
}

\section{Zusammenfassung}

Hintergrund Die Digitalisierung des Gesundheitswesens führt zu veränderten Prozessen und neuen Anforderungen. Dazu parallel, aufgrund des demografischen Wandels, steigen die gesellschaftliche Bedeutung der Pflege und damit die Erwartungen an den Einsatz von Technologie für die Gesundheitsversorgung. Neben einigen futuristisch anmutenden Ansätzen technischer Lösungen, ist ein Tablet zur Nutzung in der Biografiearbeit ein bereits tatsächlich eingesetztes Gerät.

Ziel Diese Arbeit geht der Frage nach, wie Betreuungskräfte von Menschen mit Demenz ein Tablet einschätzen, dass sie bereits regulär für die Biografiearbeit einsetzen.

Methode Es wurden teilstrukturierte Interviews durchgeführt und qualitativ interpretiert. Alle Betreuungseinrichtungen nutzten ein aus ihren eigenen Finanzmitteln gekauftes „Betreuertablet“ der Firma media4care.

Ergebnisse Vier Betreuungskräfte in drei verschiedene Einrichtungen wurden interviewt. Mediale Inhalte auf dem Tablet in Form von Videos, Bildern und Musik werden in Einzel- und Gruppenbetreuung genutzt. Berichtet wird, dass der Einsatz des Tablets bei den Bewohnern als Erzählstimulus wirkt und die Biografien dadurch mit Informationen aus erster Hand angereichert werden konnten. Das Gerät wird in den Einrichtungen nicht als alleinige Beschäftigungsmöglichkeit, sondern als Ergänzung zum bisherigen Angebot gesehen. Die betreuenden Personen wünschen sich teilweise eine ruhigere Umgebung.

Schlussfolgerungen Die Geräte wurden von den Befragten als für ihre Praxis tauglich wahrgenommen und wurde von den Bewohnern gut angenommen. Strukturierte intensive Schulungen zu dem Gerät fanden nicht statt, werden aber von allen Teilnehmenden gewünscht.

Schlüsselwörter Biografiearbeit · Assistive Technologie · Pflege · Demenz · Interview · Qualitative Forschung

Christian Buhtz

christian.buhtz@medizin.uni-halle.de

1 Gesundheits- und Pflegewissenschaften, Medizinische Fakultät der Martin-Luther-Universität Halle-Wittenberg, Dorothea Erxleben Lernzentrum Halle (DELH), Projekt FORMAT, Magdeburger Straße 12, 06112 Halle (Saale), Deutschland

2 Internationale Graduiertenakademie, Institut für Gesundheits- und Pflegewissenschaft, Medizinische Fakultät, Martin-Luther-Universität Halle-Wittenberg, Halle, Deutschland

3 Westsächsische Hochschule Zwickau, University of Applied Sciences, Zwickau, Deutschland 
Tablet-supported biography work for elderly in nursing homes: An interview study with nursing staff

\section{Abstract}

Background The digitalization of the healthcare system leads to changed processes and new requirements. At the same time, due to demographic change the social importance of care is increasing and with it the expectations for the use of technology in health care. In addition to some futuristically apparent approaches to technical solutions, a tablet for use in biography work is a device that is already in actual use.

Aim This study investigated how caregivers of people with dementia assess a tablet that they already use regularly for biography work.

Method Semistructured interviews were conducted and qualitatively interpreted. All care facilities used a caregiver tablet from the company media4care, which was purchased from their own financial resources.

Results In this study four caregivers in three different institutions were interviewed. Media content on the tablet in the form of videos, pictures and music is used in individual and group care. It is reported that the use of the tablet acts as a narrative stimulus for the residents and that the biographies were enriched with first-hand information. The device is not seen in the facilities as a sole employment opportunity but rather as a supplement to the existing offer. Some of the persons caring for the residents would like a quieter environment.

Conclusion The devices were perceived by the respondents as suitable for their practice and were well accepted by the residents. Structured intensive training on the device did not take place but was requested by all participants.

Keywords Biography work $\cdot$ Assistive technology $\cdot$ Nursing care $\cdot$ Dementia $\cdot$ Interview $\cdot$ Qualitative research

\section{Einleitung}

Die Digitalisierung löst in vielen Lebensbereichen einen Wandel aus. Das Gesundheits- und Sozialwesen im Allgemeinen und der Pflegesektor im Speziellen widmen sich, mit Ausnahme einiger Leuchtturmprojekte, erst anfänglich dieser Entwicklung (DAA-Stiftung Bildung und Beruf 2017). Die konkreten Potenziale sind folglich in der Praxis noch schwer ablesbar. Parallel hierzu nimmt die gesellschaftliche Bedeutung der pflegerischen Versorgung aufgrund der Auswirkungen des demografischen Wandels und des Fachkräftemangels spürbar zu: Die steigende Lebenserwartung und Verschiebung von Altersstrukturen zählen zu den wesentlichen Entwicklungen (Rothgang und Müller 2018; Grünheid 2015). Die Chancen, die die Digitalisierung für die pflegerische Versorgung mit sich bringt, wie beispielsweise Entlastung und Unterstützung des Pflegeprozesses, müssen demnach verstärk im Kontext der Pflegepraxis reflektiert werden (Rösler et al. 2018). Dies beinhaltet auch Reflexion über die Technikkompetenz des Betreuungspersonals als Nutzende und die Vermittlung von notwendigen Anwendungskompetenzen (Buhtz et al. 2020).

Als ein prominentes Beispiel für die Digitalisierung im Gesundheitswesen ist das Krankenhausinformationssystem (KIS) beispielhaft anzuführen, in dem Patientendaten für alle beteiligten Akteure vorgehalten und verarbeitet werden (Haas 2005; Meißner 2017). Als praktisches Beispiel ist an KIS auch zu sehen, dass Digitalisierung kein Selbstläufer ist. Die Implementierung in der Praxis ist nur erfolgreich, wenn die Nutzer von der Nützlichkeit des Systems über- zeugt sind (Gagnon et al. 2012). Für diese Überzeugungsarbeit ist mehr nötig als nur eine klassische Einweisung in ein Gerät oder eine Software. Am Beispiel KIS wird auch deutlich, dass Digitalisierung kein neues Thema ist, da diese Systeme bereits seit den 1970er-Jahren in Kliniken genutzt werden.

Grundsätzlich ist der Einsatz von Technik in der Pflege kein neues Phänomen, sondern reicht bis in die Anfangszeit der professionellen Pflege zurück (Friesacher 2010). Der Begriff der assistiven Technologie lässt sich im derzeitigen wissenschaftlichen Diskurs nicht eindeutig von früheren technischen Entwicklungen abgrenzen, wird zuweilen auch different verwendet, obgleich die Fachwelt eine konkrete Definition anstrebt (Lutze et al. 2019). Meißner geht davon aus, dass "neuartige Technologie“ zu einem eigenen Akteur in der Pflegebeziehung wird und entsprechend auf diese Beziehung einwirkt (2017). Lutze et al. stellen in ihrer aktuellen Bestandsaufnahme zur Nutzen- und Wirksamkeitsbewertung von assistiven Technologien fest, dass eine gleichberechtigte Interaktion zwischen Gerät und pflegebedürftiger Person stattfindet und Letztere die Kontrolle besitzt (2019). Die Unterstützung durch das Gerät erfolgt somit nur durch ausdrückliches (Ein-)Verständnis und die Anweisungen der pflegebedürftigen Person. Als zusätzliche Ebene in den Definitionsbestrebungen wird die Organisation beschrieben, welche sich in Wechselwirkung mit den Nutzern und den Entwicklern gegenseitig ,konfigurieren“ (Deisner et al. 2018).

Tablets, die für die Biografiearbeit eingesetzt werden und dafür entsprechend spezialisierte Software nutzen, sind eine Form von ,assistiver Technologie“. Die Biografiearbeit 
hilft, die Vergangenheit des älteren Menschen neu zu bewerten, deren Verhalten und Reaktionen in der Gegenwart besser zu verstehen und die Zukunft, sprich die Pflegemaßnahmen, entsprechend individuell zu planen (Clarke 2000). Durch die verbesserte Kenntnis der Umstände und Strukturen, welche die Person geformt haben, ist ein umfassenderes Verständnis ihrer Wünsche und Sehnsüchte möglich. Neben der gesprächsorientierten Biografiearbeit wird die Person bei der aktivitätsorientierten Variante zur Erinnerung und zum Erzählen angeregt, indem beispielsweise Bilder aus der vergangenen Zeit betrachtet oder Lieder angehört oder gar selbst gesungen werden.

In einer sich noch in der Auswertung befindlichen Cluster-randomisierten Studie, die ein selbstentwickeltes Tablet zur Biografiearbeit einsetzt, deuten erste Ergebnisse auf eine Verbesserung der Ergebnisparameter Glück, Stimmung und Sozialverhalten hin (Nordheim et al. 2017; Lech et al. 2019). Eine weitere explorative Studie, bei der ein Tablet zur Biografierecherche und -erinnerung in 6 Einzelsitzungen je Teilnehmer eingesetzt wurde, konnte die Zunahme der Zufriedenheit der Teilnehmer und eine Abnahme des Problemverhaltens beobachten (Dlugosch 2015). Beide Studien geben Indizien für die positive Wirkung von Tablet-gestützter Biografiearbeit. Jedoch konzentrieren sie sich in der Erhebung und gewählten Methodik weniger auf die Technologie und Nutzung des Geräts, sondern auf die Wirkungen der Biografiearbeit. Welchen Anteil ein Tablet bzw. dessen Software als Medium an der Therapiewirkung hat, ist aus den Arbeiten nicht abzuleiten.

Astell et al. vergleichen in ihrer Arbeit, die 2 Teilstudien umfasst, ein selbstentwickeltes Touchscreen-System (CIRCA) mit regulärer, also nichttechnikgestützter, Biografiearbeit und berichten bei CIRCA deutlich positivere Effekte bei den Bewohnern mit einer Alzheimer-Erkrankung und auch bei den Betreuenden (2008). Die Bewohner ergreifen häufiger und früher die Initiative und übernehmen auch dauerhafter die „Leitung“ einer Sitzung, wobei die Betreuenden ein dementsprechend geringeres $\mathrm{Ma} ß$ an Kontrolle und Führung ausüben müssen und wegen des gleichzeitigen Erfolgserlebnisses eine höhere Zufriedenheit mit ihrer Tätigkeit empfinden.

Eine Gruppe Forschender um Alexander Bejan von der Hochschule Furtwangen beschäftigt sich in dem Projekt „RemeMTI“, das wiederum aus dem Projekt „InterMEM“ hervorging, nicht nur mit den Effekten, sondern auch mit der technischen Entwicklung von Anwendungen für die digitale Biografiearbeit, insbesondere mit Aspekten der Bedienbarkeit und des Nutzerverständnisses für die spezielle Nutzergruppe der an Demenz erkrankten Bewohner von Pflegeeinrichtungen (Bejan et al. 2019; Bejan 2020; Bejan et al. 2018b). Dazu ergänzend wird der Algorithmus „MemoRec" entwickelt, der, basierend auf Daten einer individuellen Biografie, eine Vorauswahl über mediale Inhalte als geeignete Stimuli für die Biografiearbeit trifft und das betreuende Personal damit nicht nur unterstützt, sondern auch entlastet (Bejan et al. 2018a).

\section{Zielsetzung und Fragestellung}

Wie schätzen Betreuungskräfte von Menschen mit Demenz die Nutzung eines produktreifen und bereits in den Markt eingeführten Tablets für die Biografiearbeit ein, nachdem sie das Gerät schon längere Zeit in der regulären Versorgung eingesetzt haben? Die Betrachtung fokussiert dabei auf die Betreuer und Bewohner als Nutzer, deren Reaktion und Auswirkungen auf die Abläufe in der Betreuungseinrichtung. Aus methodischen und ethischen Gesichtspunkten ist die Untersuchung nicht ausschließlich auf Menschen mit Demenz eingegrenzt.

\section{Methode}

\section{Interview und Datenauswertung}

Für die Erhebung wurde ein qualitatives Untersuchungsdesign in Form eines teilstrukturierten Interviews gewählt (Döring und Bortz 2016).

Auf Grundlage einer Recherche zur Akzeptanz im Kontext der Technologieanwendung wurde ein Interviewleitfaden entwickelt, der durch die Konsultation zweier Forscher aus dem Bereich Pflegewissenschaft sowohl aus inhaltlicher als auch aus methodischer Perspektive qualitativ abgesichert wurde (Helfferich 2011). Weil die Befragung im Rahmen einer Qualifikationsarbeit stattfand, wurde mit Blick auf die begrenzten Ressourcen kein Pretest durchgeführt.

Der Interviewleitfaden war dreigeteilt. Der erste Teil bezog sich auf die Betreuungskraft und deren Eindrücke und Erfahrungen im Umgang mit dem Tablet. Der zweite Fragenkomplex fokussierte auf die Interaktion der Bewohner mit dem Tablet und die von den Betreuern beobachteten Auswirkungen und Reaktionen. Die Bewohner selbst wurden nicht befragt oder beobachtet. Es handelt sich also um die erinnerten Bewertungen der befragten Betreuungskräfte. Der dritte Fragenteil beschäftigte sich mit der langfristigen und weiteren Nutzung des Tablets für die Biografiearbeit sowie die Integration in die Abläufe der jeweiligen Betreuungseinrichtung und den Schulungsbedarf.

Die Interviews wurden von M.W. moderiert und die Audioaufzeichnung anschließend transkribiert. Das Datenmaterial wurde qualitativ interpretativ analysiert (Flick 2016). Dabei lag das gegenstandserkundende Erkenntnisinteresse im Mittelpunkt, und es wurden Kategorien interpretativ abgleitet. Um diese umfänglich diskutieren zu können, studierten C.B. und M.W. das vorliegende Datenmaterial in 
Abb. 1 Hauptmenü im Betreuertablet von media4care. (Eigene Darstellung)

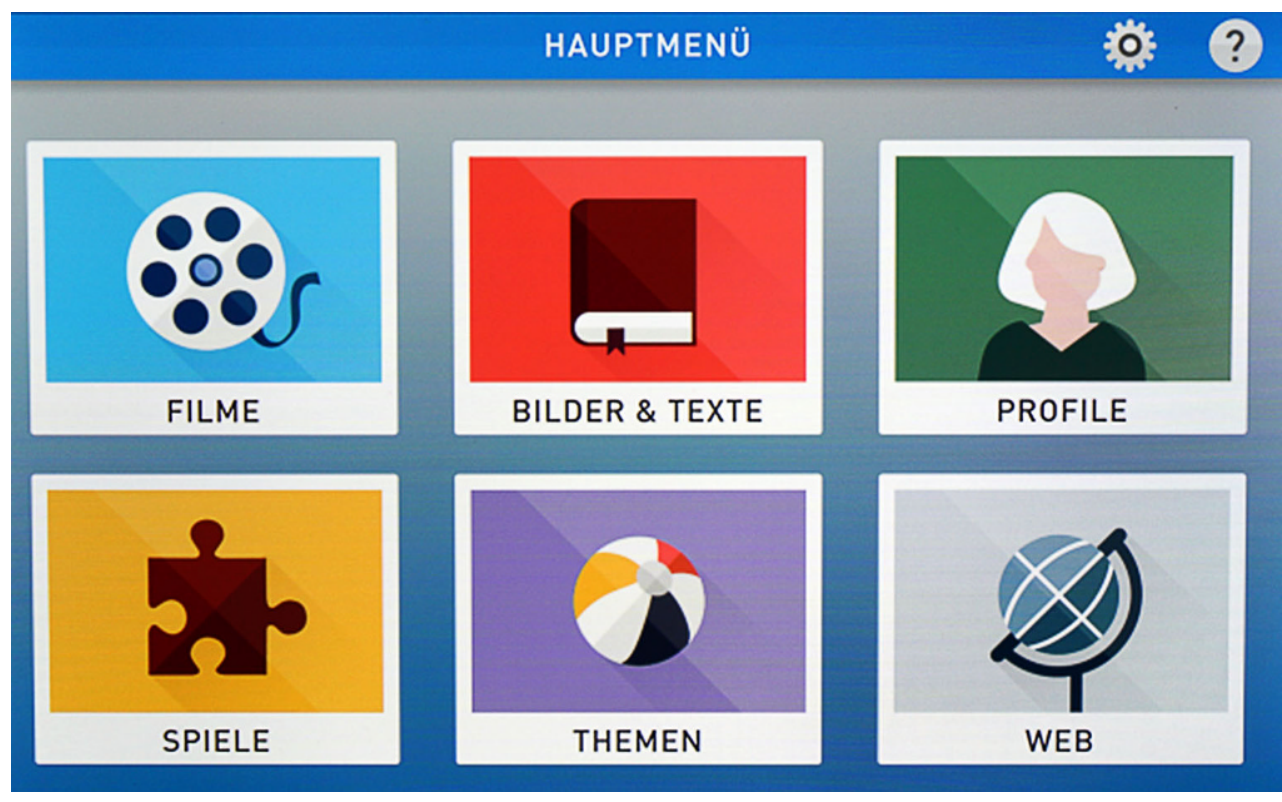

mehreren Iterationsschleifen fallbezogen, um so das Verständnis des Textes auszubauen und Bedeutungen erschließen zu können. Anschließend fand die fallübergreifende Auswertung statt, bei der zentrale Themen und Aspekte in einem induktiv erstellten Kategoriensystem dargestellt werden (Döring und Bortz 2016). Die Aufbereitung der Ergebnisse in diesem Manuskript erfolgte nach dem COREQStatement (Tong et al. 2007).

\section{Zugang zum Feld}

Das „Betreuertablet““ war den Autoren bekannt, da dies dem Projekt FORMAT als Leihgabe von der Fa. media4care zur Verfügung gestellt wurde. Die Untersuchung erfolgte ohne Kenntnis des Herstellers. Der Hersteller nennt in einer frei zugänglichen Broschüre diverse Pflegeeinrichtungen als Partner. Diese wurden von M.W. mit der Bitte um Zusammenarbeit kontaktiert. Somit wurde der Kontakt zu der KORIAN-Gruppe hergestellt, die zum Zeitpunkt der Untersuchung 10 Betreuungseinrichtungen in Sachsen betrieb. Nach einem ersten telefonischen Kontakt wurden 3 Einrichtungen identifiziert, die das Tablet bereits einsetzen. Nach der Übermittlung weiterer Informationen zum Forschungsvorhaben willigten diese ein, an den Interviews teilzunehmen. Leitende Betreuungskräfte sollten interviewt werden, da diese verantwortlich für die Einführung des Tablets waren und entsprechend einen Gesamteindruck des Einführungsprozesses und der beteiligten Kolleginnen und Bewohner besitzen. Es handelt sich bei den Interviewten somit um ein ,convenience sample“. Die Auswahl und Anzahl der Teilnehmer wurden durch die Art des Feldzugangs, also die Kontaktmöglichkeit zu den Pflegeeinrichtungen, begrenzt und festgelegt.

\section{Das Tablet}

Die Fa. media4care entwickelte ein Tablet speziell für Betreuungskräfte und Angehörige zur erleichterten Betreuung von Menschen mit Demenz. Das Tablet enthält eine Sammlung an demenzgerechten Medien, die in der Einzelund Gruppenbetreuung eingesetzt werden können (Abb. 1). Es wird keine Internetverbindung benötigt. Die Funktionen und Medien gliedern sich in die Kategorien Filme, Bücher, Spiele, Themen, Web und Profile. Die Inhalte der einzelnen Kategorien sind noch einmal thematisch gegliedert. Benutzerdefinierte Inhalte können über eine zusätzliche Speicherkarte und über einen Internetzugang ergänzt werden. Des Weiteren lassen sich individuelle Profile erstellen, welche die Biografiearbeit unterstützen, indem personenspezifische Inhalte angeboten werden können. Die Spiele sind als Text-, Foto-, Geräusch-, Musik- und Rechenrätsel gestaltet und beinhalten auch Aspekte der Problembewältigung und kreatives Gestalten.

\section{Ergebnisse}

\section{Teilnehmer und Einrichtungen}

Die Interviews fanden im Juli 2018 jeweils in den Einrichtungen vor Ort statt. Es wurden 4 Personen in 3 Einrichtungen interviewt. Die 3 Interview-Sitzungen dauerten 12, 16 und 19 min. Eine gute Gesprächsatmosphäre konnte aufgrund der bekannten Umgebung gewährleistet werden. Ein narrativer Erzählfluss wurde jedoch nur bedingt erreicht. Das Gespräch war meist von binären Fragen (FrageAntwort) geprägt. Alle teilnehmenden Personen sind weib- 
lichen Geschlechts. Zwei Interviews wurden mit je einer Person geführt, die beide leitende Betreuungskräfte waren. Beim dritten Interview war die leitende Person kurzfristig verhindert, weshalb hier ersatzweise 2 Betreuungskräfte teilgenommen haben. Die Qualifizierung des Betreuungspersonals in den Einrichtungen bewegt sich branchenüblich zwischen Betreuungsassistenten mit wenigen Wochen Ausbildung und Altenpflegekräften mit 3-jähriger Berufsausbildung. Die Anzahl der Bewohner wurde von den 3 Einrichtungen mit 59, 80 und 130 angegeben. Anderslautend zur ursprünglichen Forschungsfrage wurde bei den Bewohnerinnen und Bewohnern nicht auf ein demenzielles Krankheitsbild eingegrenzt, worauf in der Diskussion vertieft eingegangen wird.

\section{Zugang zum Gerät}

Die Angaben der Interviewteilnehmer zu der Frage, wie sie zu dem Tablet gekommen sind und wie lange sie dieses schon einsetzen, sind vage. Zwei Einrichtungen hatten nicht näher erläuterten Kontakt mit dem Hersteller und eine 14-tägige Testphase, nach der sie das Gerät dann dauerhaft angeschafft haben. Eine Einrichtung erwähnt explizit ein Mietmodel. Die andere Einrichtung beschaffte direkt ein zweites Gerät. Die dritte Einrichtung konnte keine genaueren Angaben zur Beschaffung machen. Die Nutzungszeit zum Zeitpunkt des Interviews wurde in allen 3 Einrichtungen ebenfalls ungenau mit 1 bis 2 Jahren angegeben.

\section{Kategorie: Bedienungsformen}

Das Tablet wird in den Einrichtungen überwiegend in der Einzelbetreuung, aber auch in der Gruppenbetreuung verwendet. Mit entsprechender Vorbereitung und Unterstützung bedienen einige Bewohner das Gerät selbstständig. „Manche bedienen, wenn es eingeschalten ist und das Programm eingestellt ist, das Ding selber“ (I3, 21 f). Eine Eingrenzung der Nutzung auf ein bestimmtes Krankheitsbild, also nur Bewohner mit Demenz, findet durch das Personal nicht statt. Die Gründe dafür wurden im Rahmen des Interviews nicht weiter hinterfragt. Die Nachfrage zur Nutzung des Tablets scheint das Angebot zu übersteigen. „Es ist bei uns immer gut auf die Wohnbereiche verteilt (lacht), also man hat wirklich zu tun, dass man es mal abkriegt“" (I2, 230f).

\section{Kategorie: genutzte Funktionen des Tablets}

Das gesamte audiovisuelle Angebot des Tablets wird genutzt: Videos, Bilder, Musik. Auch die Spiele wie das Rätselraten werden verwendet, wobei allerdings analogen Rätselkarten der Vorzug gegeben wurde. ,[...] die Rätselkarten ein bisschen besser, weil es gibt durchaus welche, die das noch lesen können, und wo man dann durchaus die Rätselkarte auch über den Tisch reicht und die das vorlesen, und mit dem Tablet ist es nicht ganz so [...]“ (I3, 112-114). Die Möglichkeit, das Tablet an ein TV-Gerät als vergrößertes Display anzuschließen, wird von einer Einrichtung in der Gruppensitzung verwendet. Besonders gerne genutzt werden Musik und Tierbilder. „Hab ihr Katzenbilder gezeigt, da hat sie angefangen mit lachen" (I3, 148).

Die Möglichkeit, für jeden Bewohner ein eigenes Nutzerprofil anzulegen und individuelles Bild- und Tonmaterial zu hinterlegen, wird in der Einzelbetreuung genutzt. Die vom Hersteller vorgefertigten Inhalte werden ebenfalls eingesetzt.

\section{Kategorie: Reaktionen der Bewohner}

Es wurde mehrfach eine beruhigende Wirkung auf die Bewohner berichtet: ,[...] die haben die klassische Musik zum Teil gern, das mache ich mit denen viel, die liegen. Das fördert meinetwegen, wenn sie aufgeregt sind, dann werden die ruhiger oder schlafen auch mal dabei ein. Da kannst du die ein wenig runterbringen“ (I3, 216-219).

Bei der Biografiearbeit eingesetzt, regt die Nutzung die Bewohner dazu an, über sich zu erzählen und auch miteinander ins Gespräch zu kommen. „Bewohner kommen damit sehr gut zurecht und können sich damit auch identifizieren, weil es wirklich auf die jeweiligen Lebenszonen abgestimmt ist. Seien es die Lieder, seien es irgendwelche Bilder erkennen, wo die dann aus der Vergangenheit erzählen“ (I1, 24-26).

Die Geräte scheinen teilweise auch die Möglichkeit zu eröffnen, die Beschäftigung der Bewohner zu intensivieren. „Ich kann die damit intensiver und länger beschäftigen, als wenn ich [...] animiere dazu, aus ihrer Vergangenheit zu erzählen, wo ich dann meistens anfangen muss, wo sie dann einsteigen können. So sehen sie die Bilder, lachen und fangen dann an zu erzählen“ (I1, 50-53).

Ebenso wird die aktivierende Wirkung des Tablets bei Bewohnern mit fortgeschrittenen demenziellen Symptomen beschrieben: „Bewohner, die nicht mehr ähm auf uns reagieren. [...] manche können es gar nicht mehr und starren nur noch an die Decke [...] dann merkt man dann, wenn man jetzt mit so einem Tablet hier arbeitet [...] Manche haben gerne Blumen gemocht, manche Tiere oder Verreisen und wenn man dann gerade mal so ein Video vorführt und das dem Bewohner zeigt, [...] Wenn ich erzähl, dann gucken die mich an, ja, aber sie schauen dann auch wieder zurück auf das Bild und verfolgen das regelrecht. Also sie sind so richtig mal weg und genießen das mal und machen mal kurz die Augen zu und dann gucken sie ,Ach ist noch an, ja kann ich weitergucken““”(I2, 13-24). 


\section{Kategorie: Reaktionen der Betreuerinnen}

Den Betreuungskräften macht die Arbeit mit dem Gerät Spaß, auch weil sie dadurch positives Feedback von den Bewohnern bekommen: „macht auch [...] für die Betreuungskräfte ähm viel Spaß, damit zu arbeiten, das muss man auch ganz einfach dazu sagen. Weil die dann die Freude sehen, wenn die Augen aufgehen und wie man die Dementen ganz anders mit einbeziehen kann in den Alltag" (I1, 184-187). „Also ich muss ehrlich sagen, so was hab ich schon in meiner alten Arbeitsstelle vermisst“ (I2, 273f).

Einige Betreuungskräfte scheinen eine Distanz zwischen sich und dem Bewohner zu empfinden, wenn sie das Gerät nutzen: „Es gibt Kollegen, die nehmen es nicht ganz so gerne, weil die wollen lieber am Bewohner mehr tun" (I2, 236f).

\section{Kategorie: Auswirkung auf Arbeitsabläufe}

Das Gerät unterstützt die Biografiearbeit dahingehend, dass es die Bewohner animiert, selbst etwas aus ihrer Vergangenheit zu erzählen. Diese Erzählungen haben häufig einen anderen Gehalt als die der Angehörigen. „Und dann erzählen die mitunter viel mehr, was uns persönlich aussagekräftiger ist, als wenn es Angehörige machen. Angehörige kennen das mitunter nicht mehr so" (I1, 122f).

Das Tablet wird nicht als alleinige Beschäftigungsmöglichkeit betrachtet. „Erleichtern nicht, bereichern würde ich sagen. Man kann das mal nutzen. Erleichtern [...] also keiner will, ich sage jetzt mal so, immer mit dem Tablet [...] beschäftigt werden jetzt. [...] Es muss schon Abwechslung her. Die wollen auch persönlich jetzt, sag ich mal, das man persönlich mit ihnen spricht, oder auch die Rätselkarten“ (I3, 101-105).

\section{Kategorie: Anforderung an das Umfeld, die Umgebung und Organisation}

Für die Beschäftigungssitzungen mit dem Gerät wird der Wunsch nach einer ruhigen nichtstörenden Umgebung geäußert: „Also da sollte es schon ruhig sein, aber das sollte es ähm bei Dementen in der Betreuung oder Beschäftigung allgemein sein“ (I1, 76f). Auch abgegrenzte Räumlichkeiten werden erwähnt: „Auf den Wohnbereichen oben ist es etwas schwierig, weil wir haben keine Tür, die wir zumachen können, und da ist die Lärmbelästigung schon manchmal sehr intensiv" (I2, 121-123).

Die Frage, ob Schulungen oder Workshops gewünscht sind, wurde in allen 3 Interviews positiv beantwortet. „Könnte ich mir gut vorstellen, ganz einfach auch aus dem Grund, [...] Ich kann meine Sache machen und wenn ich mal nicht weiterkomme, dann geh ich zu meiner Tochter [...] Ich wäre froh, wenn es so was gäbe, dass man mit solchen Sachen vielleicht auch mal besser zurechtkommt" (I3, 297-301).

Der Wunsch des Ausprobierens unter Anleitung und der entsprechenden Zeit und des Raums dafür wird ebenso zum Ausdruck gebracht: ,[...] überhaupt mal so auch ausprobieren und Zeit zu haben, [...] die ganze Sache mal zu machen. [...] klar für mich war das auch so, wo du (guckt zu B1) mir das gezeigt hast. [...] dann hab ich mich erst mal in die Ecke gesetzt“ (I3, 306-310).

\section{Kategorie: Probleme bei der Nutzung des Tablets}

Die Bedienung des Geräts per Touchscreen in der Anfangsphase wurde als ungewohnt für ungeübte Nutzer beschrieben. Die Verbindung mit dem Internet wird als Hürde empfunden: ,ans Internet hab ich mich nicht herangewagt bisher, also das weiß ich nicht, wie man da reinkommt, keine Ahnung" (I2, 156f).

Die Helligkeit beim Abspielen von Videos wurde in einem Fall auch moniert: ,sind zum Teil auch sehr dunkel gehalten. [...] (sucht Video auf Tablet) Wenn das irgendwie machbar ist, dass das besser vom Kontrast her ist [...] das hat die eine Bewohnerin sehr gerne gehört, da hat die auch immer geschmunzelt ne, aber gesehen hat sie dann nicht viel“" (I3, 242-244).

\section{Diskussion}

In Interviews wurden Betreuungskräfte von 3 Pflegeeinrichtungen zu ihren Erfahrungen im Umgang mit einem speziell für die Biografiearbeit entwickelten Tablet der Fa. media4care befragt. Der Schwerpunkt der Erzählung lag dabei, neben den eigenen Reaktionen, auf denen der Bewohner der Einrichtungen sowie auf den Auswirkungen auf die Arbeitsabläufe. Ein hervorzuhebendes Merkmal dieser Untersuchung ist, dass die Geräte unabhängig von dieser Studie bereits seit über einem Jahre in den Einrichtungen im Einsatz waren.

In der Literatur und Forschung wird Biografiearbeit primär im Kontext von Demenzerkrankungen thematisiert. Dennoch fand, anderslautend zur ursprünglichen Forschungsfrage, in dieser Arbeit keine explizite Eingrenzung der Tablet-Nutzung auf Menschen mit Demenz statt; nicht durch die Forschenden und auch nicht durch die Betreuenden. Es wäre ethisch kritisch zu diskutieren, wenn das Tablet nur für eine bestimmte Personengruppe ,reserviert“ würde und andere von der Nutzung ausgeschlossen wären.

Das Tablet wurde von den Betreuungskräften und Bewohnern insgesamt gut angenommen. Konkrete negative Reaktionen, Berührungsängste oder Ablehnung wurden nicht berichtet. Dies war zu erwarten, da die Einrichtungen schon vor der Untersuchung längere Zeit das Gerät nutzten 
und somit bereits eine Phase der Gewöhnung stattgefunden hatte. Obwohl dadurch die Glaubwürdigkeit der Aussagen kritisch zu hinterfragen ist, deckt sich dieses Ergebnis mit ähnlichen Untersuchungen (Bejan et al. 2018b; Astell et al. 2008; Bejan 2020). Neben der positiven Grundhaltung der Befragten wurde durchaus kritisch thematisiert, dass die Nutzung des Tablets die persönliche Zuwendung reduzieren könnte und auch nur eine Ergänzung, aber kein Ersatz zu bestehenden Beschäftigungsangebotenen sein darf.

Die Bewohner wurden nicht direkt befragt oder bei der Nutzung des Geräts beobachtet. Es gibt jedoch Aussagen von den Betreuungskräften zu den Reaktionen der Bewohner. Die beschriebenen Szenarien sind die Beruhigung von Bewohnern in angespannten Situationen, die Aktivierung von Bewohnern mit fortgeschrittenen demenziellen Symptomen und entsprechend der Biografiearbeit der Einsatz des Tablets als Erinnerungs- und Erzählstimulus.

Das betreuende Personal empfindet die positiven Reaktionen der Bewohner als Erfolgserlebnis und zieht ebenso auch den Vergleich zu einem früheren Arbeitgeber, bei dem so ein Tablet nicht zur Verfügung stand. Es ist anzunehmen, dass der Einsatz des Tablets in diesem Fall eine positive Wirkung auf die Arbeitszufriedenheit und die Attraktivität des Arbeitgebers haben kann.

Andererseits besteht die Herausforderung bei durch ein Tablet gestützter Erinnerungsarbeit auch darin, eine geeignete bzw. ruhige Umgebung zu schaffen und die Bedienung zu erlernen. So wurde auch der Wunsch nach Schulungen in allen Interviews geäußert. Beispielsweise wurde die Möglichkeit der Internetverbindung nicht genutzt, da Unklarheit darüber bestand, wie diese einzurichten sei.

Ob die Tablet-Technologie, im Vergleich zu den klassischen und analogen Medien, einen besonderen Effekt auf die Biografiearbeit besitzt, wurde nicht untersucht. Auch Dlugosch (2015) und Nordheim et al. (2017) haben den Anteil der Tablet-Technologie an der Wirkung der Biografiearbeit nicht explizit untersucht, diskutieren jedoch einige technikbezogene Aspekte. In Abgrenzung zur mit klassischen bzw. analogen Medien gestützten Biografiearbeit bemerkt Dlugosch die Möglichkeit bewegter Videobilder und die Handlichkeit sowie die an jedem Ort verfügbaren bewohnerspezifischen Inhalte (2015). Weiterhin wird das Internet als schnelle zusätzliche Informationsquelle während der Therapiesitzungen erwähnt, jedoch nicht berichtet, ob diese Funktion genutzt wurde (Dlugosch 2015). Beobachtet, aber nicht systematisch erfasst, wurden auch eine höhere Motivation und Neugier der Bewohner, vermutlich ausgelöst durch die Neuartigkeit des Mediums (Dlugosch 2015). Dass multiple Medienformen und Funktionen in einem Gerät vereint sind, hat das Potenzial, das verfügbare Angebot für die Nutzer, Bewohner und Betreuenden gleichermaßen zu verbessern, und bietet einen Mehrwert (Nordheim et al. 2017; Bejan 2020).
Darüber, inwiefern die Betreuenden oder Bewohner bestimmte Funktionen favorisieren, kann keine Aussage getroffen werden, da keine quantitativen Daten dazu vorliegen. Die beschriebenen Einsatzszenarien (Einzel- und Gruppensitzungen), wie auch die Nutzung von Musik und Videos, deckt sich mit ähnlichen Untersuchungen (Bejan et al. 2018b; Astell et al. 2008).

$\mathrm{Ob}$ und wie die Tablets in die Organisation eingefügt werden, bleibt leider offen. Es wäre von Interesse gewesen, ob es einen festen Personenkreis gibt, der für die Wartung des Geräts und die Einweisung zuständig ist. Die bereits beschriebene „Hürde“ des Internetzugangs würde mit einer technisch betreuenden Person oder einer Einweisung nicht mehr als Hindernis wahrgenommen.

Die Planung und Durchführung der Studie waren ressourcenmäßig eingeschränkt, da es sich um eine Qualifikationsarbeit auf Bachelor-Niveau handelte, die nicht alle relevanten Aspekte berücksichtigen konnte. Somit war eine fortlaufende Befragung bis zur theoretischen Sättigung des Datenmaterials nicht realisierbar. Ein paralleles Kodieren der Interviews und ein dadurch ständig angepasster Interviewleitfaden hätten den Gehalt der Daten möglicherweise noch gesteigert. Die Anzahl der Interviews ist ebenso durch die Rahmung als Bachelor-Arbeit begründet.

Dass, mit Ausnahmen, nur Leitungskräfte für die Interviews zur Verfügung standen, ist mit methodischen Vorüberlegungen zu begründen, die retrospektiv kritisch zu betrachten sind. Es wäre wünschenswert gewesen, mehr Personen und auch eine breitere Auswahl an Qualifizierungsstufen im Sample präsent zu haben. Es ist grundsätzlich sehr schwierig, Betreuungspersonal neben seiner beruflichen Tätigkeit zusätzlich für Forschungsvorhaben zu akquirieren. Dies deckt sich mit Erfahrungen der Autoren dieser Arbeit aus anderen Feldstudien mit Pflegefachpersonen und Altenpflegekräften.

\section{Stärken und Limitationen}

Die Stärke der Untersuchung besteht darin, dass die Befragung unabhängig vom Hersteller des Tablets und unabhängig von dem Prozess der Geräteeinführung in den Einrichtungen stattfand. Alle Einrichtungen haben aus von der Forschergruppe unabhängigem Antrieb heraus Interesse für das Tablet entwickelt und es in ihren Einrichtungen eingeführt.

Die in der ursprünglichen Fragestellung stattfindende Eingrenzung auf Menschen mit Demenz als Nutzende des Tablets bzw. Bewohner der Pflegeeinrichtungen ließ sich in der praktischen Durchführung nicht realisieren und wäre, wie bereits im vorherigen Abschnitt diskutiert, ethisch kritisch zu werten. 
Das gewählte qualitative Untersuchungsdesign, worin die leitfadengestützten Interviews gezielt auf das Forschungsziel eingehen konnten, erwies sich als adäquat. Aussagen zu Effekten oder Wirksamkeit können nicht getroffen werden. Eine Generalisierbarkeit der Ergebnisse war entsprechend dem qualitativen Ansatz nicht das Ziel. Ebenso stellt die vorliegende Studie den Zustand zu einem bestimmten Zeitpunkt dar. Eine Aussage über den Verlauf und die damit einhergehende Entwicklung kann nicht getroffen werden. Eine Sättigung der Daten wurde aufgrund der geringen Anzahl der Interviewteilnehmer nicht erreicht, was die Aussagekraft der Ergebnisse begrenzt, jedoch im Kontext der vorliegenden Literatur tendenzielle Aussagen zulässt.

Die Interviewteilnehmer hatten in den Einrichtungen leitende Funktionen oder wurden von leitendem Personen für das Interview ausgewählt. Eine Verzerrung der Berichte hin zum Positiven kann daher nicht ausgeschlossen werden. Dieses Risiko bezieht sich insbesondere auf die Berichte über die Reaktionen der Bewohner, da diese Berichte nicht auf eigenen, sondern auf erinnerten Beobachtungen Dritter, den Interviewteilnehmerinnen, beruhen.

\section{Schlussfolgerung}

Die vorliegende Erhebung zeigt, dass die durch ein spezialisiertes Tablet gestützte Biografiearbeit in den 3 hier untersuchten Betreuungseinrichtungen bereits regulär eingesetzt und genutzt wird sowie auch als geeignet betrachtet wird.

Obgleich die Grundhaltung und Erfahrungen der Befragten durchweg positiv sind, steht die Anwendung des Tablets nicht für sich allein, sondern ist in ein Betreuungskonzept einzubetten. Persönliche Gespräche und Zuwendung sollen nicht substituiert, sondern angeregt werden. Auch die Beschäftigung mit nichttechnischen Artefakten, wie beispielsweise „Rätselkarten“, oder körperliche Aktivitäten sind nicht weniger von Bedeutung.

Jedoch stellt es auch neue Anforderungen an das Personal und die Organisation. Die Wartung des Gerätes muss sichergestellt sein. Ausgewählte Mitarbeiter sollten als Expertinnen und Experten bzw. Multiplikatoren fungieren, ansprechbar sein und nicht nur bei Problemen mit dem Gerät helfen, sondern auch adäquate Schulungen, die über eine simple Geräteeinweisung hinausgehen, für die Kolleginnen und Kollegen anbieten (Rösler et al. 2018; Buhtz et al. 2020). Hier würden sich u. a. akademisierte Pflegefachpersonen anbieten. Eine in der Einrichtung für das Gerät zentrale zuständige Person könnte nicht nur Wissen und Kompetenzen für die Nutzung eines Geräts an die Mitarbeiterinnen und Mitarbeiter vermitteln, sondern zugleich auch in Richtung des Herstellers pflegefachlich begründete Anfor- derungen kommunizieren und am Weiterentwicklungsprozess partizipieren.

Die Vermittlung von Wissen und Kompetenzen an die nutzenden Mitarbeiter darf hier nicht nur auf die Gerätebedienung an sich abzielen, sondern muss die Betreuungskräfte dazu befähigen, für jeden Bewohner selbstständig, über die bedarfsgerechte (Nicht)Nutzung des Geräts entscheiden zu können. Wesentlich für die Annahme und den sozialverträglichen sowie mehrwertbringenden Einsatz neuer Technologie ist eine positive Grundhaltung der Nutzer. Diese kann durch adäquate Bildungsangebote in Form von Schulungen, Workshops, Kollegen als Multiplikatoren und die Partizipation am Entwicklungsprozess erreicht werden.

Es kann vermutet werden, dass ein derart erweitertes Aufgaben- und Kompetenzprofil das berufliche Selbstbewusstsein hebt, die Attraktivität des Arbeitsplatzes steigert und somit langfristig auch der Akquise und der Bindung von Personal dient.

Danksagung Die Autoren bedanken sich bei den Interviewteilnehmern für ihre Mitarbeit sowie bei der KORIAN-Gruppe als Betreiber der Betreuungseinrichtungen und bei media4care als Hersteller des Tablets für die Zusammenarbeit.

Förderung Dieses Manuskript entstand im Rahmen des Forschungsprojekts FORMAT gefördert durch das Land Sachsen-Anhalt. Das Projekt ist Teil des Forschungsverbunds „Autonomie im Alter“. Das in dieser Untersuchung verwendete Tablet wurde von der Herstellerfirma media4care an das Projekts FORMAT für die Dauer der Projektlaufzeit ausgeliehen. Die Leihgabe geschah ohne Zweckbindung oder andere Verpflichtungen.

Funding Open Access funding enabled and organized by Projekt DEAL.

Interessenkonflikt C. Buhtz, D. Paulicke, M. Wolf, S. Hofstetter und K. Schwarz geben an, dass kein Interessenkonflikt besteht.

Open Access Dieser Artikel wird unter der Creative Commons Namensnennung 4.0 International Lizenz veröffentlicht, welche die Nutzung, Vervielfältigung, Bearbeitung, Verbreitung und Wiedergabe in jeglichem Medium und Format erlaubt, sofern Sie den/die ursprünglichen Autor(en) und die Quelle ordnungsgemäß nennen, einen Link zur Creative Commons Lizenz beifügen und angeben, ob Änderungen vorgenommen wurden.

Die in diesem Artikel enthaltenen Bilder und sonstiges Drittmaterial unterliegen ebenfalls der genannten Creative Commons Lizenz, sofern sich aus der Abbildungslegende nichts anderes ergibt. Sofern das betreffende Material nicht unter der genannten Creative Commons Lizenz steht und die betreffende Handlung nicht nach gesetzlichen Vorschriften erlaubt ist, ist für die oben aufgeführten Weiterverwendungen des Materials die Einwilligung des jeweiligen Rechteinhabers einzuholen.

Weitere Details zur Lizenz entnehmen Sie bitte der Lizenzinformation auf http://creativecommons.org/licenses/by/4.0/deed.de.

\section{Literatur}

Astell AJ, Alm N, Gowans G, Ellis MP, Dye R, Campbell J (2008) CIRCA: a communication prosthesis for dementia. In: Mihaili- 
dis A, Boger J, Kautz HA (Hrsg) Technology and aging. IOS Press, Amsterdam, S 67-76

Bejan A (2020) Rekindling autobiographical memories of people with dementia via digital technology - the RemeMTI project. Hyve Health Well Being. https://nbn-resolving.org/urn:nbn:de:bsz:fn1opus4-63273

Bejan A, Gündogdu R, Butz K, Müller N, Kunze C, König P (2018b) Using multimedia information and communication technology (ICT) to provide added value to reminiscence therapy for people with dementia; lessons learned fromthree field studies. Z Gerontol Geriat 51:9-15. https://doi.org/10.1007/s00391-017-1347-7

Bejan A, König P, Kirchhofer B, Kunze C, Kienzler R (2019) Schlussbericht INTERMEM : INTERMEM : Projekt InterMem - Technikgestützte Biografiearbeit und Erinnerungspflege : Laufzeit des Vorhabens: 06/2015 bis 09/2018

Bejan A, Plotzky C, Kunze C (2018a) Memorec-towards a lifetheme-based reminiscence content recommendation system for people with dementia. In: Miesenberger K, Kouroupetroglou G (Hrsg) Computers helping people with special needs. Springer, Cham, S 509-513

Buhtz C, Paulicke D, Hofstetter S, Jahn P (2020) Technikaffinität und Fortbildungsinteresse von Auszubildenden der Pflegefachberufe; Eine Online-Befragung. HBScience. https://doi.org/10.1007/ s16024-020-00337-5

Clarke A (2000) Using biography to enhance the nursing care of older people. Br J Nurs 9:429-433. https://doi.org/10.12968/bjon.2000. 9.7.6323

DAA-Stiftung Bildung und Beruf (2017) Digitalisierung und Technisierung der Pflege in Deutschland; Aktuelle Trends und ihre Folgewirkungen auf Arbeitsorganisation, Beschäftigung und Qualifizierung. https://www.input-consulting.de/files/inpcon-DATA/ download/2017_digitalisierung_und_technisierung_der_pflege_ 2.pdf. Zugegriffen: 12. Mai 2018

Deisner J, Hergesell J, Maibaum A (2018) Nutzerkonfiguration und konfigurierende Nutzer in ambulanten Pflegesettings. In: Weidner R, Karafillidis A (Hrsg) Dritte Transdisziplinäre Konferenz „Technische Unterstützungssysteme, die die Menschen wirklich wollen“, S 25-33

Dlugosch P (2015) Tabletgestützte biografische Spurensuche fördert die Erinnerungsleistung bei Demenzerkrankten. NeuroGeriatrie 12:61-68

Döring N, Bortz J (2016) Forschungsmethoden und Evaluation in den Sozial- und Humanwissenschaften. Springer, Berlin, Heidelberg

Flick U (2016) Qualitative Sozialforschung; Eine Einführung. Rowohlt, Reinbek bei Hamburg
Friesacher H (2010) Pflege und Technik; eine kritische Analyse. Pflege Ges 15:293-313

Gagnon M-P, Desmartis M, Labrecque M, Car J, Pagliari C, Pluye P, Frémont P, Gagnon J, Tremblay N, Légaré F (2012) Systematic review of factors influencing the adoption of information and communication technologies by healthcare professionals. J Med Syst 36:241-277. https://doi.org/10.1007/s10916-010-9473-4

Grünheid E (2015) Regionale Aspekte des demografischen Wandels. https://www.bib.bund.de/Publikation/2015/Regionale-Aspektedes-demografischen-Wandels.html. Zugegriffen: 17. Dez. 2018

Haas P (2005) Medizinische Informationssysteme und elektronische Krankenakten. Springer, Berlin

Helfferich C (2011) Die Qualität qualitativer Daten; Manual für die Durchführung qualitativer Interviews. Springer VS, Wiesbaden

Lech S, O'Sullivan JL, Gellert P, Voigt-Antons J-N, Greinacher R, Nordheim J (2019) Tablet-based outpatient care for people with dementia. GeroPsych 32:135-144. https://doi.org/10.1024/16629647/a000210

Lutze M, Glock G, Stubbe J, Paulicke D (2019) Digitalisierung und Pflegebedürftigkeit; Nutzen und Potenziale von Assistenztechnologien. CW Haarfeld, GmbH

Meißner A (2017) Technisierung der professionellen Pflege. Einfluss. Wirkung. Veränderung. In: Hagemann T (Hrsg) Gestaltung des Sozial- und Gesundheitswesens im Zeitalter von Digitalisierung und technischer Assistenz. Nomos, Baden-Baden, S 155-171

Nordheim J, O'Sullivan J, Gellert P, Arndt S, Kuhlmey A, Antons J (2017) Tablet-based intervention for nursing home residents with dementia: a cluster Rct. Innov Aging 1:1370. https://doi.org/10. 1093/geroni/igx004.5041

Rösler U, Schmidt K, Merda M, Melzer M (2018) Digitalisierung in der Pflege; Wie intelligente Technologien die Arbeit professionell Pflegender verändern. https://www.inqa.de/SharedDocs/PDFs/ DE/Publikationen/pflege-4.0.pdf?_blob=publicationFile\&v=2 . Zugegriffen: 18. Juli 2018

Rothgang H, Müller R (2018) BARMER Pflegereport 2018. BARMER. https://www.barmer.de/blob/170372/ 9186b971babc3f80267fc329d65f8e5e/data/dl-pflegereportkomplett.pdf. Zugegriffen: 11. Dez. 2018

Tong A, Sainsbury P, Craig J (2007) Consolidated criteria for reporting qualitative research (COREQ): a 32-item checklist for interviews and focus groups. Int J Qual Health Care 19:349-357. https://doi. org/10.1093/intqhe/mzm042

Hinweis des Verlags Der Verlag bleibt in Hinblick auf geografische Zuordnungen und Gebietsbezeichnungen in veröffentlichten Karten und Institutsadressen neutral. 